\title{
Das Herz der Welt \\ Hohlwelt-Theorien in der Literatur-, Wissens- und Konspirationsgeschichte
}

Der folgende Beitrag legt gewissermaßen eine case study zu einem einst unnützen, zumindest nicht verifizierbaren Wissensnarrativ vor, das als metaphorischer Thesaurus andere Wissenstraditionen (Religion, Naturphilosophie, Literatur) alimentierte, bis es diese Leistung an dafür besser geeignete literarische Formen - wie etwa die Science-Fiction - abgeben musste. Von aller naturwissenschaftlichen, sozialen und anthropologischen Zuständigkeit abgezogen, fand das frei flottierende Wissen zuletzt seinen Ort in einem obskuren konspirativen politischen Diskurs und war damit zu einem Mythos im Sinne Roland Barthes' geworden: Je mehr es seine empirische Referenz verloren hat, desto maßloser wurde sein Anspruch als Welterklärungswissen. ${ }^{1}$ So gesehen stellen meine Überlegungen keinen Beitrag zum pittoresken oder gar subversiven Charakter des Unnützen Wissens dar, sondern verweisen auf seine Risiken.

Frustriert von den etablierten Wissenschaften seiner Zeit, der Philosophie, der Jurisprudenz und auch der Theologie, sucht Faust sein Heil in der Magie, um hier eine Antwort auf seine drängende Frage zu finden, "was die Welt / im Innersten zusammenhält« ${ }^{2}{ }^{2}$ Mephistopheles ist gut beraten, nicht diese Frage zu beantworten, sondern lieber exquisite sinnliche Genüsse und Erfahrungen in Aussicht zu stellen, um Faust zur Vertragsunterschrift zu bewegen. Denn im Horizont der zu Fausts Zeiten dominierenden Formen spekulativen Wissens - etwa des gelehrten Jesuitenpaters Athanasius Kircher $^{3}$ - hätte Mephisto auf Fausts grundlegende Frage nur eine enttäuschende Antwort geben können: Nichts. Denn was die Welt im Innersten zusammenhält, ist nach Überzeugung maßgeblicher Autoritäten des 17. und auch noch des 18. Jahrhunderts nur ein Vakuum, ein leerer Raum. Sogar der Pudel hat einen Kern, ${ }^{4}$ die Erde hingegen ist im Inneren hohl. Die-

Vgl. Roland Barthes, Mythen des Alltags, Frankfurt a.M. 1964.

Johann Wolfgang von Goethe, Faust. Eine Tragödie, in: Ders., Goethes Werke, hg. von Albrecht Schöne, Frankfurter Ausgabe Bd. 7.1, Frankfurt a.M. 1999, S. 34, V. $583 f$.

3 Athanasius Kircher, Mundus subterraneus (1665).

4 Vgl. Goethe, Faust, V. 1323, S. 64. 
ses Konzept von der hohlen Erde hat eine obsolete Vor- und eine dubiose Nachgeschichte, auf die ich im Folgenden, kursorisch und mit subjektiven Akzentsetzungen, eingehen möchte. Dazu unternehme ich fünf Bohrungen ins Erdinnere, die zugleich die Stationen einer Zeitreise markieren: Die erste führt in die vom Glauben beherrschte Welt des Mittelalters, die zweite in das wissenschaftsfreudige Zeitalter der Frühaufklärung, die dritte in die Epoche der Romantik, in der Literatur und Naturphilosophie als spekulative Disziplinen konkurrieren und sich ergänzen, die vierte ins 19. Jahrhundert zu Jules Verne und seinem häretischen, aber grundsoliden Wissenschaftsvertrauen, und die fünfte und letzte schließlich ins 20. Jahrhundert und in unsere Gegenwart, in der sich Gossip und Paranoia zum Hohlwelttheorem die Stichworte liefern.

\section{Das Mittelalter: Das Innere der Erde als Reich des Satans}

Als einen der ersten maßgeblichen Hohlwelttheoretiker darf man wohl Dante bezeichnen. Er lokalisiert die Hölle im Inneren der Erde, wo man schon in der Antike das Reich der Toten vermutet hatte. Der Tartarus war so tief, dass ein hinabgeworfener Amboss neun Tage und Nächte fiel, bis er aufschlug. ${ }^{5}$ Bei Dante entstand der Höllenkrater beim Aufprall des aus dem Himmel gestürzten Luzifers. In neun gewaltigen, sich nach unten zunehmend verjüngenden, terrassenförmig angeordneten Ringen erstreckt sich seither das Reich des Bösen, in dem die Sünder büßen.

Der erste Kreis besitzt ungeheure Ausmaße, um all jene aufnehmen zu können, die als ehrenwerte Heiden - wie Homer, Horaz usf. - vor der Zeit Christi lebten, also nicht erlöst werden konnten. Ab dem zweiten Höllenkreis beginnt das Reich der Qualen, wo »Heulen und Zähneklappern herrschen« (Mt 8,12): Hier werden die Wollüstigen gepeinigt, im dritten die Schlemmer. Der vierte Kreis widmet sich denen, deren Charakter zu Geiz, Maßlosigkeit oder Trägheit neigte, der fünfte versammelt die zornigen Seelen. Im sechsten Kreis schließlich büßen Ketzer und Gottlose, im siebten die Gewalttätigen, also Mörder und Selbstmörder, aber auch Räuber und Sodomiten; im achten die Kuppler, Huren, Schmeichler und Fälscher. Im neunten Kreis schließlich, im Mittelpunkt der Erde, eingezwängt in einer

5 Vgl. dazu Umberto Eco, Die Geschichte der legendären Länder und Städte, München 2013, S. 348. 
Eisplatte, sitzt Luzifer selbst, zusammen mit den Schlimmsten der Schlimmen, den Verrätern Brutus, Cassius und Judas.

In den einzelnen Abteilungen der unterschiedlichen Höllenkreise sind der Teufel und seine Spießgesellen - deren Zahl der gelehrte Dämonologe Jean Wier auf nicht weniger als 7405926 Teufel beziffert hat (nämlich 72 Höllenfürsten zuzüglich 1111 Legionen zu je 6666 Dämonen) ${ }^{6}$ - damit beschäftigt, die Sünder zu martern. Hohlweltreisende, die bei Gelegenheit einer Jenseitsschau auch einen Blick in die Hölle werfen durften, berichten davon, dass der am Grunde des Kraters platzierte Satan die ihm von oben gereichten Sünder einverleibt und wieder defäkiert. Sobald der Seelenkot auf die Eisfläche prallt, nehmen die Sünder wieder ihre ursprüngliche Gestalt an und gebären - das gilt für Männer gleichermaßen wie für Frauen unter grauenhaften Schmerzen monströse Kreaturen, die sie von innen her zerreißen. Dann beginnen ihre Qualen von Neuem. Theresa von Ávila, die Gott gebeten hatte, ihr zum Zweck religiöser Erbauung einen Einblick in die Hölle zu gewähren, befiel noch viele Jahre nach diesem Gnadenerweis, sobald sie an ihre Schau zurückdachte, am ganzen Leibe ein Zittern. ${ }^{7}$ Nicht einmal der Teufel findet Vergnügen an der Schinderei: Dante beschreibt, wie das dreimäulige, abscheuliche Ungeheuer, während es sich die Sünder einverleibt und ausscheidet, zugleich aus sechs Augen weint. ${ }^{8}$

Die Berichte der Jenseitsreisenden konvergieren durchweg im Befund, dass in der unterirdischen Welt eine ordo inversa herrscht, die dem "himmlischen Jerusalem« ein »infernalisches Babylon" gegenüberstelle ${ }^{9}$ - ein Reich des Grams und der Düsterkeit, aus dem das Glück für immer vertrieben ist. Die Natur kennt hier keinen Frieden, nur den Zustand des Aufbegehrens: Eisstürme und Feuerwalzen, Dürre und peitschender Regen, tobende Winde und heulenden Sturm. Die Pflanzen, die hier wachsen, »strotzen vor Dornen und tragen Früchte voll giftigen Saftes. ${ }^{10}$

Man muss das Ausmaß der Trostlosigkeit ermessen, das in die Höllendarstellungen der Literatur und Kunst eingegangen ist, um den Trotz würdigen zu können, mit dem der Herrscher dieser düsteren Welt sich zu seiner Apostasie bekennt: In John Miltons Paradise Lost (1667) trägt der Teufel

\footnotetext{
Vgl. dazu Johann Wier, De praestigïs Daemonum, Basel 1563.

Vgl. Arturo Graf, Satan, Beelzebub und Luzifer. Der Teufel in der Kunst, New York 2009, S. 190.

8 Vgl. Dante Alighieri, Die göttliche Komödie. Die Hölle, hg. von Erwin Laaths, Wiesbaden o.J., XXXIV, V. 1265-1321.

9 Vgl. Arturo Graf, Satan, Beelzebub und Luzifer, S. 172.

10 Ebd., S. 177.
} 
das Höllenfeuer, in dem die Sünder ihre Vergehen büßen, selbst in seiner Brust: "The Hell within him, for within him hell / he brings.«" Die Qualen, die Luzifer anderen bereitet, erträgt er auch selbst, aber er imponiert dabei durch seinen titanischen Trotz: "Better to reign in Hell than serve in Heav'n ${ }^{12}{ }^{2}$ Dieser Luzifer, der blanke Geist der Rebellion, kann daher zur Berufungsinstanz der Gothic Novel werden, jener literarischen Gattung, in der das Aufklärungszeitalter seinem allzu hochgestimmten Optimismus die düstere Anthropologie nachträgt. ${ }^{13}$

In der Spannbreite der Höllen- und Hohlweltdarstellung zwischen dem infernalischen Folterkeller, wie ihn die Literatur und die bildende Kunst des christlichen Mittelalters, und dem glanzvoll-düsteren Palast von Eblis, wie ihn der Schauerroman beschrieben hat, ${ }^{14}$ ist bereits die Ambivalenz aller hohlwelttheoretischen Modelle beschlossen: Im Inneren der Erde herrscht das Grauen, aber auch das Geheimnis. Die Finsternis dunkelt ein, aber sie bietet auch Schutz. Vor allem aber ist das Innere der Erde ein Raum ungeheurer Kräfte, mächtiger Energien, eines Rumorens, das noch die Oberfläche erzittern macht: Beben und Vulkanausbrüche sind die äußeren Symptome eines Aufruhrs im Inneren, wo das Feuer rast und die Seelen der Verdammten stöhnen. Die Hölle, die Heimat Satans und der Unterweltgötter, ist auch der Ort der Gegenwelt, die am Sturz der oberen Ordnung arbeitet, die unterminiert, Fallen stellt und auf ihre Stunde wartet. Diese Idee von den buchstäblich subversiven Kräften unter der Erdoberfläche findet noch eine späte Resonanz in jenen Abenteuergeschichten, wo die Höhlen unter der Erde und die Welt der Minen und Schächte zum Schutzraum der Besiegten werden, wo sie ihre Kräfte sammeln und ihre Rückkehr vorbereiten können, man denke an den eingekerkerten Edmond Dantès in seiner Höhle im Château d'If in Alexandre Dumas' Der Graf von Monte Christo, an Rudolf von Gerolstein in Eugène Sues Les Mystères de Paris oder an Jean Valjean in Hugos Les Misérables. Noch Harry Lime, der Verbrecherkönig des Penicillinhandels, hat in der Kanalisation im Nachkriegswien, wie es Graham Greene und Carol Reed im Dritten Mann beschreiben, seinen für andere unerreichbaren Zufluchtsort.

11 John Milton, Das verlorene Paradies, Werke Englisch-Deutsch, Frankfurt a.M. 2000, S. 168.

12 Ebd., I. 263, S. 36.

13 Vgl. dazu Ingeborg Weber, Der englische Schauerroman - eine Einführung, München 1983; Mario Grizelj (Hg.), Der Schauer(roman). Diskurszusammenhänge - Funktionen Formen, Würzburg 2010.

14 Etwa in William Beckfords Roman Vathek, an Arabian Tale or The History of the Caliph Vathek, 1786, wo der titelgebende Prinz den Palast des Teufels besucht. 


\section{Frühe Neuzeit und Frühaufklärung: Die Geheimnisse des Magnetismus}

Das Innere der Erde, das so lange von Religion und Folklore als Höllenreich angesehen wurde, wird in der frühen Moderne zum Gegenstand einer wissenschaftlichen Spekulation über seine physikalische Beschaffenheit. Diese Diskussion wurde maßgeblich von dem englischen Astronom Sir Edmund Halley, der auch dem geschweiften Kometen seinen Namen gegeben hat, inspiriert. Zwar haben die meisten Wissenschaftler dieser Zeit sich auf die bis heute gültige Vorstellung eines heißen Erdinnern mit glühendem Kern geeinigt, aber Halleys Theorie, die er in einem Vortrag vor der Royal Society 1691 darlegte, demzufolge drei konzentrische Hohlkugeln im Innern der Erde durch ihre Bewegung für den Magnetismus der Pole verantwortlich seien, fand Resonanz bei durchaus namhaften Wissenschaftlern wie dem Schweizer Mathematiker Leonhard Euler und dem englischen Physiker Sir John Leslie. Mit Halleys Hypothese wurde die theoretische Grundlage für die vielen literarischen Expeditionen ins hohle Erdinnere gelegt, die bis auf den heutigen Tage nicht nur in der Populärkultur zum Gegenstand intensiv und kontrovers diskutierter explorativer Phantasmen wurden. ${ }^{15}$

15 Eine Auswahl wichtiger Romane (R), Erzählungen (Erz.), Traktate (Tr.) und Pamphlete (Pamphl.) zur Hohlwelt: 1720: Simon Tyssot de Patot, La vie, les aventures et le voyage de Groenland du réverend Père Cordelier Pierre de Mésange (R.); 1721: Anonymus, Relation d'un voyage du pôle arctique au pôle antartique par le centre du monde (R.); 1735: Charles de Fieux Chevalier de Mouhy, Lamékis, ou les voyages extraordinaires d'un Egyptien dans la terre intérieure (R.); 1742: Ludvig Holberg, Nicolai Klims unterirdische Reise (R.); 1787: Giacomo Casanova, Icosameron ou Histoire d'Eduard et d'Elisabeth (R.); 1820: Captain Adam Seaborn (d.i. Cleves Symmes), Symzonia. A Voyage of Discovery (R.); 1821: Collin de Plancy, Voyage au centre de la Terre, ou Aventures diverses de Clarancy et des ses compagnons dans le Spitzberg, au pôle Nord et dans les pays inconnues (R.); 1828: Anonymus, Die Unterwelt oder Gründe für ein bewohnbares und bewohntes Inneres unserer Erde (Dr.); 1833: Edgar Allan Poe, Ms. Found in a Bottle (Erz.); 1835: Edgar Allan Poe, The Unparalleled Adventure of One Hans Pfaall (Erz.); 1837: Edgar Allan Poe, The Narrative of Arthur Gordon Pym of Nantucket (R.); 1841: Edgar Allan Poe, A Descent into the Maelström (Erz.); 1852: Alexandre Dumas Père, Isaac Laquedem (R., Fragm.); 1863: René de Pont-Jest, La Tête de Mimer (R.); 1864/67: Jules Verne, Voyage au centre de la terre (R.); 1865: George Sand, Laura. Voyage dans le crystal (R.); 1868: William F. Lyon, The Hollow Globe (Pamphl.); 1871: Edward BulwerLytton, The Coming Race (R.); 1894: Henry M. Howell, The cosmic problem solved, and paradise found (Tr.); 1899: Cyrus Teed/Ulysses G. Morror, The cellular Cosmogony, or, The earth a concave sphere (Tr.); 1908: George Emerson Wells, The Smokey God (R.); 1915: Edgar Rice Burroughs, At the Earth's core (R.); 1929: Léon Creux, Le Voyage de l'Isabella au centre de la terre (R.); 1984: Miguel Serrano, Adolf Hitler, el ultimo avatar (Pamphl.); 1989: Jean Robin, Opération Orth, ou l'incroyable secret de Rennes-leChateau (Pamphl.); Umberto Eco, Die Geschichte der legendären Länder, verweist auf 
Immer geht es in diesen Texten - zumeist umfangreichen Romanen - um wagemutige Forschungsreisende, die durch eine Erdspalte fallen oder auf See von einen schweren Sturm weitab nach Norden oder Süden getrieben werden und dann in einen tosenden Wasserstrudel geraten, der sie geradewegs an einem Pol ins Innere der Erde hineinführt und oft auch zum anderen Ende wieder hinaus. Im Inneren der Welt entdecken die Reisenden zu ihrem Erstaunen meist hochentwickelte Zivilisationen, oft Nachkommen der Bewohner der hyperboreischen Länder des äußersten Nordens, aber auch des untergegangenen Atlantis oder der verschollenen Stämme Israels. Der Aufenthalt unter der Erde gibt den Reisenden Anlass zu ethnologischen Studien und staatstheoretischen Überlegungen, die häufig auch Kritik an den engstirnigen religiösen und sozialen Verhältnissen der Welt über Tage erlauben, etwa im lateinisch verfassten Roman Nicolai Klims unterirdische Reise des Dänen Ludvig Holberg. Hier landet der Held nach dem obligaten Sturz in eine Erdspalte auf dem unterirdischen Planeten Lazar, wo er Völker von unterschiedlichem Zivilisationsgrad kennenlernt, wobei die höchstentwickelten zugleich die umgänglichsten sind, die keiner autoritären Gesetzgebung bedürfen. $\mathrm{Zu}$ solchen tendenziell satirischen Indienstnahmen des Themas, die nicht selten auch utopische Entwürfe bzw. dystopische Warnungen begünstigen, zählt auch der 1787 erschienene, fünfbändige Roman Icosameron ou Histoire d'Eduard et d'Elisabeth aus der Feder des berühmt-berüchtigten Giacomo Casanova. Hier berichten die alt gewordenen Geschwister Eduard und Elizabeth, achtzig Jahre nach ihrem geheimnisvollen Verschwinden, wie sie auf einer Schiffahrt in den Norden vom Malstrom verschlungen und in einer wasserdichten Kiste ins Innere der Erde gelangten. Dort treffen sie auf eine Gesellschaft ätherischer, androgyner Wesen, die Megamikren, deren Philosophie und Lebensweise Casanova detailliert beschreibt. ${ }^{16}$

Was all diese - literarisch nur selten aufregenden - Texte verbindet, ist über die Vermutung von der Erde als einem Hohlkörper hinaus auch die Idee von im Inneren der Erde lebenden Populationen, die nicht selten der menschlichen Rasse überlegen sind. Zu dieser Spekulation konnte es dank

eine ganze Reihe weiterer Romane und auf mehrere Bibliographien, die zusätzliche Texte namhaft machen: Guy Costes/Joseph Altairac, Les terres creuses, Paris 2006; Cynthia Ward, "Hollow Earth Fiction", in: The internet review of Science Fiction, www.irosf. com/q/zine/article/10460 (2008) [Datum des letzten Zugriffs: 09.02.2015.].

16 Eco berichtet, dass Casanovas ermüdend langweiliger Roman erfolglos blieb. Die Druckkosten verschlangen das karge Vermögen des an seinem Lebensabend als Bibliothekar in Böhmen tätigen Abenteurers. Vgl. Umberto Eco, Die Geschichte der legendären Länder, S. 355 . 
einer weiteren theoretischen Annahme kommen, die sich unter Geophysikern und Philosophen im 18. Jahrhundert einer großen Beliebtheit erfreute. Demzufolge befand sich die im Winkel von 23,5 Grad geneigte Erdachse ursprünglich lotrecht zu der elliptischen Bahn der Erde um die Sonne - erst eine kosmische Katastrophe führte zu der heutigen Neigung. Diese Katastrophe brachte aber gewaltige Überschwemmungen, Veränderungen des Klimas und eben auch eine Verlagerung der Pole mit sich. Die Vorstellung eines goldenen Zeitalters und die mit diesem Gedanken assoziierten Mythen und Narrative von untergegangenen Welten ließen sich so nicht nur erklären, sondern auch noch zu geschichtsphilosophischen Utopien ausweiten: Phantasien von blühenden Landschaften im Erdinneren, in die andere Wesen oder sogar humanoide Zivilisationen, seien es nun die ersten Exemplare des Homo sapiens, seien es die Ur-Arier, seien es Vertreter früherer Rassen, sich haben retten können, werden zu einer Hoffnung der vom Untergang bedrohten Welt. Eine restitutio ad integrum kann so als Ziel einer geheimen Weltordnung der Drohung der Kontingenz entgegengestellt werden.

Für keinen Geringeren als Jean-Jacques Rousseau, den vielleicht bedeutendsten Sohn der Stadt Genf, war die Achsenverschiebung gewissermaßen der Startmechanismus für die fatale zivilisatorische Entwicklung. In seinem Essai sur l'origine des langues von 1761 heißt es:

Stellen Sie sich einen immerwährenden Frühling im Lande vor, überall Wasser, Vieh und Weiden. Stellen Sie sich dazwischen Menschen vor, wie die Natur sie geformt hat; ich wüsste nicht, weshalb sie je auf ihre primitive Freiheit hätten verzichten und das isolierte Hirtenleben hätten verlassen sollen, das ihrer natürlichen Schwerfälligkeit so angemessen war, um sich ohne Not Sklaverei aufzuerlegen, Arbeit und Schwierigkeiten, die von einem Leben in Gesellschaft nicht zu trennen sind.

Derjenige, welcher wünschte, dass der Mensch gesellig werde, berührte mit dem Finger die Achse des Globus und neigte ihn zur Achse des Universums. Durch diese kleine Bewegung sehe ich das Antlitz der Erde verändert und das Geschick der menschlichen Rasse entschieden. Von ferne höre ich die Freudenschreie der unwissenden Menge, sehe Paläste und Städte entstehen und die Künste erblühen, die Gesetze und den Handel; ich sehe die Völker sich bilden, sich aufösen und ineinanderfolgen wie die Fluten des Meeres, ich sehe Menschen, die sich in verschiedenen Gegenden in deren Ansiedlungen zusammenballen, um sich gegenseitig zu zerfleischen und aus der übrigen Welt eine entsetzliche Wüste zu machen, ein würdiges Denkmal der sozialen Verbindung und der Nützlichkeit von Künsten und Wissenschaften. ${ }^{17}$

17 Jean-Jacques Rousseau, Essay über den Ursprung der Sprachen, worin auch über Melodie und musikalische Nachahmung gesprochen wird, in: Ders., Musik und Sprache, 
Die Achsenverschiebung also ist nach Rousseau verantwortlich für die Fehlentwicklung des menschlichen Geschlechts vom friedlichen Hirten- und Nomadendasein zu einem Leben in Verstellung, Heuchelei und ewigem Zwist. Es ist naheliegend, dass im mentalen Klima von Spätaufklärung und der Empfindsamkeit, als deren Exponent Rousseau den Konventionen der zivilen Gesellschaft den Gehorsam aufkündigte, Vorstellungen eines ursprünglichen, unverdorbenen Menschseins mit der aufgeklärten Hoffnung auf die Perfektibilität von Mensch und Gesellschaft kollidierten und dass in literarischen Texten wie den oben genannten der Konflikt zwischen beiden, den Perfektibilisten der Aufklärung und den Träumern vom goldenen Zeitalter, auf den imaginären Reisen der Literatur ausgetragen werden konnte. Die Vermutung unzugänglicher Pole und einer gewissermaßen natürlichen Schieflage des Planeten entsprach einem Denken, das die Welt, wie sie ist, als die beste aller denkbaren Welten hinzunehmen willens war: die Vorstellungen von einer unnatürlichen Schieflage, von Polöffnungen und einem bewohnten inneren Kern hingegen einer Welt, wie sie einmal gewesen war und wie sie wieder werden sollte.

Was bei Rousseau und anderen wohl als eher theoretisch produktive Spekulation diente, sollte sich unter dem Druck kameralistischer und ökonomischer Interessen bald auch einer pragmatischen Prüfung zu stellen haben. Der Gedanke einer prinzipiell kolonisierbaren inneren Welt mag das Manifest des vormaligen amerikanischen Hauptmannes John Cleves Symmes inspiriert haben, der 1818 eine Fülle gelehrter Gesellschaften und den amerikanischen Kongress aufforderte, ihm eine Expedition mit einer hundertköpfigen Mannschaft zu finanzieren:

»An die ganze Welt!

Ich erkläre, dass die Erde hohl und auf der Innenseite bewohnbar ist; dass sie eine Anzahl fester konzentrischer Sphären, eine in der anderen, enthält; dass sie am Pol über 12 oder $16 \mathrm{Grad}$ offen ist; ich verpfände mein Leben zur Unterstützung dieser Wahrheit und bin bereit, den Hohlraum zu erforschen, wenn mich die Welt bei diesem Unternehmen unterstützen und mir helfen wird.«

John Cleves Symmes, aus Ohio, ehemaliger Infanteriehauptmann. ${ }^{18}$

Ausgewählte Schriften, übers. von Dorothea Gülke/Peter Gülke, Wilhelmshaven 1984, S. 99-168, hier S. 127f.

18 Zit. nach Materialien von Volker Dehs zu seiner vorzüglich und umfassend kommentierten Ausgabe von Jules Verne, Reise zum Mittelpunkt der Erde, Düsseldorf/Zürich 2005, S. 335-432, hier S. 403. 
Symmes' Vorschlag, endgültig den Nachweis zu erbringen, dass die Erde wie eine Matrjoschka-Puppe mehrere konzentrische Kugeln enthalte, die durch große Eingänge an beiden Polen miteinander verbunden seien und dem Menschen Lebensraum bieten könnten, also: Unnützes Wissen nutzbringend zu verwenden, wurde nicht ernst genommen. ${ }^{19}$ Da er mit Entdeckerfreude und Entschlusskraft nicht imponieren konnte, verlegte sich Symmes auf die Überzeugungskraft der literarischen Imagination. Zwei Jahre nach seinem Aufruf, 1820, erscheint unter dem Namen eines Captain Adam Seaborn (hinter dem sich wohl kein anderer als Cleves Symmes verbarg) der Roman Symzonia. A Voyage of Discovery, der die Reise des Ich-Erzählers über die Falkland-Inseln und den Südpol zu einem unterirdischen Volk von großer Weisheit, den Symzonianern, beschreibt, deren Lebensgewohnheiten ausführlich geschildert werden. ${ }^{20}$ Trotz dieser literarischen Intervention bleibt Symmes' Wunsch nach einer Expedition unerfüllt - aber sein Beitrag stabilisierte die Tradition der literarischen Hohlweltreisen. Immer wieder finden sich nun nur geringfügig variierte literarische Szenarien, wonach Reisende von magnetischen Kräften am Nordpol ins Innere der Erde gezogen und von dort nach den üblichen Begegnungen mit unbekannten Völkern am Südpol wieder an die Erdoberfläche retourniert werden, wo sie einer zumeist ungläubigen Zuhörer- und Leserschaft die Vorzüge oder - seltener - die Nachteile eines Lebens unter der Erde vermitteln wollen. Fast vollständig ist nun jenes Terrain kartographiert, in das dann im 19. Jahrhundert die literarischen Entdeckungsreisen von Edgar Allan Poe und Jules Verne führen werden.

\section{Die Romantik: Von der Hochzeit der Gesteine}

Zuvor aber sind die eigentümlichen Spekulationen der deutschen Romantik zu berücksichtigen, die ein fast obsessives Interesse am Inneren der Erde entwickelt haben - dabei findet der Streit über eine hohle oder gefüllte Erde mit einem glühenden oder kalten Kern nur eine indirekte, aber umso erbittertere Resonanz in der Debatte zwischen Neptunisten und Vulkanisten, die immer-

19 Vgl. ebd., S. 351.

20 Weitere Hinweise zur Rezeptionsgeschichte von Symmes in der üppig kommentierten Neuausgabe von Edgar Allan Poe, Die Geschichte des Arthur Gordon Pym aus Nantucket, hg. von Hans Schmid/Michael Farin, Hamburg 2008. 
hin Goethe zeitweilig intensiv beschäftigte. ${ }^{21}$ Theodore Ziolkowski hat in seinem Werk über Das Amt des Poeten darauf hingewiesen, dass die Protagonisten der deutschen Romantik zum Teil beachtliche Karrieren im Bereich des Bergbaus vorweisen konnten. Zur eigentümlichen romantischen Aura des Bergbaus mögen empirische Gründe beigetragen haben wie die Tatsache, dass im industriell unterentwickelten Deutschland in den Bergwerken keine Kohle geschürft wurde, sondern wertvolle Erze. Hier prägten daher keine düsteren Abraumhalden und verrußte Arbeiter das Bild der Bergbaustädte wie in England, wo John Keats in den Kohlebergwerken noch nach der mittelalterlichen Tradition die »dunklen Fabriken Satans« zu erblicken meinte. ${ }^{22}$ In Deutschland hingegen stimulierte der farbige Anblick zünftiger Meister und ihrer Gesellen in schmucker Tracht die romantischen Dichter zu historischen Konjekturen über untergegangene Welten, zu Phantasien von den geheimnisvollen Kräften der Steine und Fossilien und zu der Überzeugung vom esoterischen Wissen derer, die sie bergen und sammeln. Die erste Bergbauakademie der Welt wurde 1765 in Freiberg gegründet und hatte lange Vorbildcharakter für andere Nationen - der hier als Lehrer tätige, charismatische Abraham Gottlieb Werner beeinflusste das Denken der romantischen Generation vielleicht nicht minder als Gotthilf Heinrich von Schubert mit seinen Ansichten von der Nachtseite der Naturwissenschaften (1808).

Ein Abstieg in den Schacht eines Bergwerks war fester Bestandteil jener romantischen Wanderungen, wie sie Tieck und Wackenroder unternahmen, die Höhlen im Berginneren wurden mit religiöser Ehrfurcht wie gotische Kathedralen beschrieben, die kundigen Bergleute wie Priester verehrt. In den Schächten der Minen wachsen - nach einem im 18. Jahrhundert noch weit verbreiteten Glauben - Steine und Metalle wie organische Materie, sie haben männlichen oder weiblichen Charakter, halten Hochzeit und zeugen Edelsteine. Unedles läutert sich im Dunkel der Erde zu Wertvollem. Dass Silber oft in bleiernem Erz gefunden wurde, galt als "Beleg für den Prozeß der Selbstvervollkommnung des Bleies über das Erz zum Silber [...]. ${ }^{23} \mathrm{We}$ gen dieser Vorstellung von der organischen Natur der steinernen Schatzkammer im Inneren der Erde bildet sich eine Ästhetik heraus, die den Inbegriff des Schönen direkt im Herzen der Finsternis erblicken konnte. Der alte böhmische Bergmann in Novalis' Ofterdingen-Roman metaphorisiert die

21 Vgl. Gerd-Rainer Riedel/Jochen Klauß/Horst Feiler, Der Neptunistenstreit. Goethes Suche nach Erkenntnis in Böhmen, Uckerland 2009.

22 Zit. nach Theodore Ziolkowski, Das Amt des Poeten, Die deutsche Romantik und ihre Institutionen, Stuttgart 1992, S. 37.

23 Ebd., S. 43. 
Metalle und Edelsteine als Blumen der Tiefe: »welches köstliche Gewachs blüht ihm auch in diesen schauerlichen Tiefen. ${ }^{24}$ An anderer Stelle gedenkt er jener sagenhaften Urzeit, "wo jeder Keim, noch einsam für sich schlummerte, und einsam und unberührt sich vergeblich sehnte, die dunkle Fülle seines unermesslichen Daseyns zu entfalten. ${ }^{25}$ Wenn Steine, Metalle und Felsformationen nach dem Glauben des Novalis und einiger seiner Zeitgenossen Dokumente der Erdgeschichte waren, so war diese Überlieferung im Sediment der Steine aufbewahrt. Wegen des organischen Charakters der Steine war auch der Zugang zum Erdinneren nicht einfach »ein kaltes, dunkles Loch [...] es war ein vitaler, pulsierender Ort, in den der Mensch hinabstieg wie in seine eigene Seele, um dort der menschlichen Erfahrung in drei wesentlichen Dimensionen zu begegnen: der Geschichte, der Religion und der Sexualität. ${ }^{26}$ Der Abstieg in den Berg vermittelt also nicht nur geologische, sondern auch historische und anthropologische Einsichten.

Insofern im Inneren der Berge die Zeit stillsteht, ist hier auch der Ort der sogenannten 'Bergentrückung, also des Glaubens, dass unter der Erde die Zeit verlangsamt oder sogar angehalten werden kann - ein weitreichender Gedanke, der von nun an die Hohlwelt-Theorien dominieren wird. Gestalten wie Tannhäuser im Hörselberg, wie Kaiser Barbarossa im Kyffhäuser, wie Friedrich II. schlafen im Inneren der Berge und warten auf ihre Erweckung, um das Volk von großen Plagen oder dem Antichrist zu befreien. Die Geschichte des Jünglings in Falun, der am Tag vor seiner Hochzeit in den Berg einfährt, dort verschüttet wird, nach 50 Jahren, in Eisenvitriol in seiner strahlenden Jugend konserviert, an den Tag gebracht, von seiner früheren Braut erkannt und sogleich zu Staub verfällt, ${ }^{27}$ sollte freilich eher als Menetekel auf chiliastische Hoffnungen gelesen werden. Auch die Besucher

24 Novalis, Schriften. Die Werke Friedrich von Hardenbergs, hg. von Paul Kluckhohn/ Richard Samuel, 4 Bde., Stuttgart 1960-1975, Bd. 1, S. $245 f$.

25 Ebd., S. 252; vgl. dazu Theodore Ziolkowski, Das Amt des Poeten, S. 45.

26 Ebd., S. 46f.

27 Die wichtigsten literarischen Bearbeitungen des wahrscheinlich durch die Erwähnung in Schuberts Ansichten von der Nachtseite der Naturwissenschaft (1808) für die deutsche Literatur so produktiv gewordenen Topos: Achim von Arnim, Des ersten Bergmanns ewige Jugend (eine Ballade aus dem Roman Armut, Reichtum, Schuld und Buße der Gräfin Dolores, [1810]); Johann Peter Hebel, Unverhofftes Wiedersehen (1811); E.T.A. Hoffmann, Die Bergwerke zu Falun (1819); Hugo von Hofmannsthal, Das Bergwerk zu Falun (1899, vertont von Rudolf Wagner-Régeny); Richard Wagner hat ein Libretto zu Die Bergwerke zu Falun (1842) geschrieben. In der Lyrik fand das Motiv Resonanz bei Friedrich Rückert, Die goldne Hochzeit; Georg Trakl, Ellis-Gedichte; Karl Bernhard von Trinius, Des Bergmanns Leiche; vgl. dazu Thomas Eicher (Hg.), Das Bergwerk von Falun. Varianten eines literarischen Stoffes, Münster 1996. 
der schlafenden Kaiser oder anderer Hohlweltbewohner, der Zwerge, Elfen, Salamander und holländischer Matrosen, werden von deren Zeitenthobenheit betroffen - denn die Dauer ihres Aufenthalts unter der Erde scheint den romantischen Wanderern, die einen Weg ins Erdinnere gefunden haben, nur ein Spiel im Augenblick zu sein, doch zurück an der Oberwelt müssen sie feststellen, Jahre ihres Lebens verloren, um Jahrzehnte gealtert zu sein - die Sage von den drei Bergleuten in Grimms Sagensammlung, Tiecks Märchennovelle Die Elfen oder Washington Irvings berühmte Geschichte von Rip van Winkle haben dem Thema der Zeitenthobenheit jeweils eigene Pointen abgewonnen. ${ }^{28}$

Die »dunklen, feuchten Stätten in der Erde, wo unablässig die Erzeugung von Steinen und Metallen vor sich geht «, ${ }^{29}$ lassen sich unschwer auch als Schauplätze einer dunklen und intensiven Erotik identifizieren. Schächte und Höhlen sind Orte nicht für traute romantische Herzensergießungen, sondern durchaus für "satyrhafte Paarungen ${ }^{30}$ und erlauben die literarische Evokation einer ekstatischen Liebeserfahrung, die sich buchstäblich untergründig, aller christlichen Bezähmung zum Trotz, erhalten hat. In Novalis' noch vergleichsweise unverdächtigem Lied vom Herm der Erde klingt jene Idee einer profunden erotischen Symbiose an, die in den romantischen Venuskultnovellen den Protagonisten zum Schicksal werden wird:

Der ist der Herr der Erde,

Wer ihre Tiefen misst,

Und jegliche Beschwerde,

In ihrem Schooß vergisst.

Wer ihrer Felsenglieder

Geheimen Bau versteht

Und unverdrossen nieder

Zu ihrer Werkstatt geht.

Er ist mit ihr verbündet

Und inniglich vertraut

Und wird von ihr entzündet,

Als wär' sie seine Braut. ${ }^{31}$

28 Vgl. dazu Hans Richard Brittnacher, Die Zeit des Zauberschlafs. Ein Motiv romantischer Erzählkunst bei Ludwig Tieck und Washington Irving, in: Athenäum, Jahrbuch für Romantik 12 (2002), S. 133-154.

29 Theodore Ziolkowski, Das Amt des Poeten, S. 64.

30 Ebd., S. 65.

31 Novalis, Schriften I, S. 247. 
Der hier noch in den Konjunktiv gesetzte Frevel - »als wär' sie seine Braut« - wird in Novellen wie Tiecks Runenberg oder E.T.A. Hoffmanns Die Bergwerke zu Falun Wirklichkeit. In den Vorstellungen der Lustgrotte, in der Tannhäuser den Liebesdienst für Venus versieht, oder jenes Lustgemachs, in dem Christian in Tiecks Runenberg die Herrin seiner Begierde erblickt, das steinerne nackte Weib, lebt die Vorstellung des Erdinneren als Ort eines erotischen Geheimnisses fort - es ist die romantische Gegenreligion, die gar nicht anders kann, als das, was sich dem Einverständnis der Zeit und ihren moralischen Konventionen entzieht, was Verlockung und Entgrenzung bereithält, hinter einem Vorhang anzusiedeln, der nur gelegentlich, für den Bruchteil eines Augenblicks, gelüftet werden kann, ${ }^{32}$ oder aber ihn tief ins Innere der Erde zu verbannen, dorthin also, wo seit alters her auch die Hölle vermutet wird. Dass so viele Helden der Romantik immer wieder dorthin einfahren, auf der Suche nach kostbarem Gestein, das in ihrer Wahrnehmung sich kaum unterscheidet vom Exzess sinnlicher Lust, ${ }^{33}$ den sie sich von Eisfeen und marmornen Statuen erhoffen, dass sie im Berginneren Erfahrungen machen, die sie auf immerdar dem Leben an der Oberwelt buchstäblich entrücken werden - das ist das Geheimnis, das dem Inneren der Erde zugesprochen wird: "Es ist die Dunkelheit des Erdreichs, worin, noch vor der Sonne geschützt, die Saat keimt. ${ }^{34}$

Kein Autor der späten Romantik hat die Versuchungen einer Reise ins Erdinnere so ostentativ perhorresziert wie Edgar Allan Poe - in mehreren seiner Erzählungen ${ }^{35}$ sowie in seinem einzigen Roman, The Narrative of Arthur Gordon Pym of N Nantucket (1834). Hier wird Symmes' Vorstellung von the holes in the poles zum Anlass einer spektakulären Untergangsphantasie. Das Motiv der Polreise erhält zudem besondere Nahrung durch das zeitgenössische Interesse an der Erschließung der Nordwestpassage zwischen dem pazifischen und dem stillen Ozean - binnen weniger Jahrzehnte, erst noch in Dekadenabständen,

32 Vgl. dazu Peter von Matt, Liebesverrat. Die Treulosen in der Literatur, München 1991, S. $184-197$.

33 Vgl. Hartmut Böhme, Romantische Adoleszenzkrisen. Zur Psychodynamik der Venuskultnovellen von Tieck, Eichendorff und E.T.A. Hoffmann, in: Text und Kritik 10 (1981), S. 133-176.

34 Rüdiger Safranski, Romantik, Eine deutsche Affäre, München 2007, S. 122.

35 In seinen Erzählungen Ms. Found in a Bottle von 1833 und A Descent into the Maelström von 1841 thematisiert Poe die Idee des Trichters am Ende der Welt. In Poes Satire The Unparalleled Adventure of One Hans Pfaall (1835) bemerkt der über die Arktis im Ballon fliegende Held, dass die Eisfläche "in an circular centre endet, "whose dusky hue, varying in intensity, was at all time darker than any other spot upon the visible hemisphere«. Edgar Allan Poe, The Complete Works, Bd. 2, hg. von James A. Harrison, New York 1979, S. 89. 
dann aber in zunehmender Beschleunigung, erscheinen nun literarische Texte, die dem Geheimnis der Pole und der toten Welt ewigen Eises nachspüren wollen. Poes Roman schildert eine buchstäbliche rite de passage in einer männlichen, von Matrosen, Meuterern und Wilden belebten Welt, in der das verdrängte Weibliche die Männer, die sich ihm entziehen wollten, zu verderben sucht. ${ }^{36}$ Immer wieder wird Arthur Pym, der Held des Romans, schiffbrüchig, immer wieder wird er gerettet, immer wieder bei lebendigem Leib begraben, immer wieder aus den Armen des Todes freigegeben, und immer wieder treibt er orientierungslos umher, um gerettet und erneut ausgesetzt und von der magnetischen Kraft des Pols angezogen zu werden. In zwei Gestalten trachtet die Natur nach ihm, dem Weißen aus der kultivierten Ostküstenstadt: in Gestalt von entsetzlichen Schwarzen oder aber in Gestalt des Ozeans, seiner mal trägen Fluten, mal aggressiven Wellen, die den Schiffbrüchigen verschlingen wollen. Überdeutlich ist die weibliche Codierung der Gefahren, der Höhlen und Bäuche, in denen die Helden gefangen gehalten werden, der Fluten, die nach ihnen greifen, der Tiergestalten, die das Wasser bewohnen. Der antarktische Lebensraum ist eben nicht kalt, sondern Ort einer mütterlichen, geradezu hypnotischen Wärme, das Wasser verliert seine klare Konsistenz, wird trübe und milchig; die Lebewesen dieser sonderbaren Fauna haben katzenhafte Gesichter, ein plüschiges weißes Fell, rote Krallen, rote Lippen und sogar rote Zähne. Die aus Rot und Weiß, dem Blut der Geburt und dem Weiß des Todes zusammengesetzten Monster bringen das archetypische Motiv jeder Polreise, den blutenden Albatros aus Coleridges Versepos vom Ancient Mariner (1798) in Erinnerung und lassen die neugierigen oder sogar frevelhaften Reisenden und Entdecker den Sündenfall der Entweihung der jungfräulichen Polgegenden durch den Sturz in den Malstrom büßen. Je mehr sich die Schiffbrüchigen dem Pol nähern, desto mehr häufen sich die Epiphanien des Weiblichen, desto milchiger wird das Wasser, desto lauter schreien die Vögel, desto kraftloser werden die Helden. Bevor sie am Ende der Welt ankommen und in einen Trichtersog geraten und über die Kante ins Erdinnere stürzen, fällt Pyms Blick auf eine monumentale weibliche Gestalt, die magna mater, die sich in ein Leichentuch gehüllt hat: »And the hue of the skin of the figure was of the perfect whiteness of the snow. « ${ }^{37}$

${ }^{36}$ Detaillierter hierzu: Hans Richard Brittnacher, Kältetod. Eismeer- und Polreisefantasien um 1800, in: Barry Murname/Andrew Cusack (Hg.), Populäre Erscheinungen. Der deutsche Schauerroman um 1800, München 2011, S. 291-308.

37 Edgar Allan Poe, Geschichte des Arthur Gordon Pym, S. 242; vgl. dazu die immer noch überzeugende Interpretation von Leslie A. Fiedler, Liebe, Sexualität und Tod. Amerika und die Frau, Frankfurt a.M./Berlin 1987, S. 312ff. 
IV. Die Entzauberung der Leere: Jules Verne und das 19. Jahrhundert

Für einen in der Wolle gewirkten Positivisten wie Jules Verne war eine solche Dämonisierung des Erdinneren unerträglich - er tritt mit seinem Roman Voyage au centre de la terre 1864 den Nachweis an, dass die Erde hohl ist, dass sie zu durchreisen ist und dass eine solches Abenteuer dem Reisenden lehrreiche Einsichten zu liefern vermag. ${ }^{38}$ Die Besichtigung des Erdinnern ist nicht nur eine Lektion in Geologie, sondern erlaubt auch einen Blick in den Bauplan der Schöpfung, der dem romantischen Schwärmer verschlossen bleiben muss. Vernes Voyages extraordinaires, so der Untertitel seiner Geschichten, meinen keine unwahrscheinlichen, sondern lediglich außergewöhnlich lehrreiche Unternehmungen, wobei das Beiwort raußergewöhnlich mehr noch als die Reiseumstände den akademischen Ertrag der Reise charakterisiert. An staatstheoretischen Diskussionen wie den früheren Hohlweltromanen liegt Verne wenig, auch seine erzählerischen Tollkühnheiten sind zuletzt von einem eisernen Vertrauen auf eine prinzipiell mögliche naturwissenschaftliche Erklärbarkeit und auch auf die technische Realisierbarkeit solcher abenteuerlicher Expeditionen geprägt - zumal die Wissenschaftler dieses Romans eine Reise nachvollziehen, die bereits 300 Jahre vor ihnen ein Alchemist ohne technische Unterstützung zurückgelegt hat. ${ }^{39}$ Die Helden suchen, nachdem ein rätselhaftes Manuskript ihre Neugierde geweckt hat, den Eingang zur Unterwelt in einem isländischen Vulkan namens Snoeffelsjökull, geraten zunehmend tiefer in die Erde, entdecken hier längst ausgestorbene Lebewesen wie Mammuts und Dinosaurier, auch riesige Menschen von vier Metern Größe, fahren über unterirdische Ozeane, durchqueren gewaltige Wälder aus Champignons und geraten schließlich, als eine Sturzflut sie zu ertränken droht, auf ein fossiliertes Floß, auf dem sie durch einen Krater wieder an die Erdoberfläche geschleudert werden. Anders als in der literarischen Tradition üblich führt ihre unterirdische Reise nicht vom Nordpol zum Südpol, sondern in eurozentrischer Verkleinerung der Erddurchquerung vom isländischen Snoeffelsjökull zur Mittelmeerinsel Stromboli. Das Zentrum des Erdinneren haben sie nicht gesehen, die Frage nach seinem kalten oder kochenden Herzen können sie zuletzt nicht entscheiden, wohl aber die Lehre einer ausgehöhlten Erde be-

38 Ausführlicher dazu Peter Schnyder, Jules Verne, Voyage au Centre de la Terre (1864/67), in: Roland Borgards/Harald Neumeyer/Nicolas Pethes/Yvonne Wübben (Hg.), Literatur und Wissen. Ein interdisziplinäres Handbuch, Stuttgart 2013, S. 375-379.

39 Vgl. ebd., S. 376. 
kräftigen, deren Ablagerungen Aufschluss geben über die Entstehung der Welt - und über die Vitalität dieser Welt, die den Vergleich mit der Welt über Tage nicht zu fürchten braucht.

Mit Verne gelangt die unschuldige Spekulationswut der Hohlwelttheoretiker an ihr Ende: Selbst die Versuchung, nach romantischem Vorbild »den Abstieg ins Innere der Erde und die anschließende Eruption als Bilder sexueller Bewusstwerdung zu deuten ${ }^{40}$, erscheint unangemessen angesichts des unerschütterlichen Positivismus des Autors und der naiven Abenteuerlust seiner Helden. Das Phantasma der hohlen Welt hat zwar neben Jules Verne im 19. Jahrhundert noch einige andere phantastische Ausprägungen erfahren - etwa im Roman Isaac Laquedem (1852) von Alexandre Dumas Père, in dem der Ewige Jude eine Reise ins Erdinnere antritt und dort, Poe hätte eine helle Freude gehabt, auf die Parzen trifft, das dreiköpfige Symbol tödlicher Weiblichkeit, denen er Kleopatras Lebensfaden abringt, oder bei George Sand, deren Roman Laura. Voyage dans le crystal (1865) in bewährter phantastischer Tradition den wissenschaftlichen Ehrgeiz der Forscher und ihre Gier nach Edelsteinen exemplarisch bestraft. Das letzte größere Beispiel dieser Tradition von Hohlweltromanen dürfte Edward Bulwer-Lyttons The Coming Race von 1871 sein, in der ein junger Mann nach einem Sturz in eine Erdspalte die unterirdische Zivilisation der Vril-ya kennenlernt, die ein elysisches Leben zu führen scheinen, bis sich dem Helden die Augen öffnen für den fatalen Konformismus einer Wohlstandsgesellschaft, in der persönlicher Mut und ästhetische Kreativität ausgestorben sind. Zu einem Zeitpunkt, als das Phantasma der hohlen Erde endgültig seine spekulative Dimension verliert und der tristen Realität der mit wissenschaftlichem Eifer betriebenen Förderung der Bodenschätze weicht, wird Jules Vernes phantastische Bekräftigung der Hohlweltlehre, die zudem vorgibt, mit der avancierten Wissenschaft im Bunde zu stehen und mit geradezu pedantischer Gründlichkeit die Behauptungen der Hohlwelttheoretiker prüft, bestätigt oder widerlegt, zu einer liebenswerten, aber skurrilen literarischen Anomalie, die jene spekulative Energie, die bis dahin das literarische Experiment mit der Hohlwelt beseelt hatte, endgültig verloren hat.

${ }^{40}$ Vgl. dazu Volker Dehs, Vernes Reise zum Mittelpunkt der Erde, Nachwort, S. 346. 


\section{Das 20. Jahrhundert: Im Griff der Paranoia.}

Spätestens bei Bulwer-Lytton ist die allegorische Dimension des Hohlweltmotivs offenbar geworden: Es geht nicht wirklich um unterirdische Zivilisationen oder gar um den möglichen Zugang zu ihnen, sondern in Wahrheit nur um ein ökonomisches Interesse an ihren materiellen Ressourcen. Die traditionelle Funktion der Metapher, den clash of cultures beim Betreten der hohlen Welt und der Begegnung mit ihren Bewohnern zum Anlass des Nachdenkens über das Leben über Tage zu nützen, wird zunehmend von den sich allmählich etablierenden Science-Fiction-Romanen übernommen. Nachdem auch geistig schwerfällige Hardliner den Unsinn einer hohlen Welt eingesehen haben, lassen sich die von first contact-Szenen angestoßenen ethnologischen und sozialtheoretischen Diskussionen sehr viel plausibler, weil weniger leicht falsifizierbar, an der Fiktion einer Begegnung mit den Populationen anderer Galaxien entwickeln. ${ }^{41}$

Der Charme der Hohlwelt-Theorien bestand in der Einladung, von einem anderen Leben zu phantasieren, ohne sich deshalb vor der Realität blamieren zu müssen. Es war eine vergleichsweise seriöse Mythenkonstruktion, die zwar hemmungslos, aber auch mit einem Augenzwinkern spekulierte. Sie blieb reizvoll, solange sie unverbindlich war. Sie hatte ihre Geltung, weil niemand es besser wissen wollte. Nachdem jedoch die wissenschaftliche und ökonomische Entwicklung das unterirdische Terrain entzauberte, nachdem die Literatur diese Desillusionierung nachvollzogen hatte, war die Hohlwelt-Theorie unwiderruflich vom Schauplatz der Diskurspraktiken und Wissensordnungen verwiesen. Mit der Pseudoplausibilität der Annahme eines phantasmatischen leeren Raums war auch das utopische Potenzial eines dort beheimateten anderen Lebens gestorben. Dem trägt die derzeit letzte Variante der Hohlwelt-Theorie Rechnung, in der das Erdinnere noch als ein unterirdisches Mausoleum für die bösen Geister der Toten dient. Miguel Serrano, ein 1917 geborener Chilene, der zeitweilig Botschafter seines Landes in Indien, Jugoslawien und Österreich war, eng befreundet mit G.G. Jung und Hermann Hesse, ${ }^{42}$ legte 1984 sein - wie er es nannte - Lebenswerk vor unter dem Titel Adolf Hitler, el ultimo avatar. ${ }^{43}$ Der Titel ist wörtlich zu verstehen, denn Serrano hält Hitler für den Zehnten Avatar

41 Vgl. dazu Roland Innerhofer, Science Fiction, in: Hans Richard Brittnacher/Markus May (Hg.), Phantastik. Ein interdisziplinäres Handbuch, Stuttgart 2013, S. 318-328.

42 Vgl. Miguel Serrano, Meine Begegnung mit C.G. Jung und Hermann Hesse in visionärer Schau, Zürich/Stuttgart 1968.

43 Ders., Adolf Hitler - Der letzte Avatar, Santiago 2004. 
von Vishnu, der menschliche Gestalt angenommen habe, um das Ende des Kali Yuga herbeizuführen. 1945, kurz vor der Kapitulation, sei Hitler durch einen von Albert Speer entworfenen unterirdischen Gang, der den Führerbunker mit dem Flughafen Tempelhof verband, entkommen und habe in einer fliegenden Untertasse Deutschland verlassen, um fortan im unterirdischen Reich des Südpols zu leben und den bislang exoterischen Krieg nun auf esoterischer Ebene weiterzuführen. ${ }^{44}$ Den Südpol schätzte Hitler als Aufenthaltsort, weil er der Garten Eden der arischen Rasse war, bevor diese durch das Kippen der Erdachse geteilt wurde. Der Pol sei keineswegs ein Ort unerträglicher Kälte, sondern ein warmes Land, auf das man stoße, sobald man einen schützenden Eissaum überwunden habe. Unterschiedliche Gruppen - der CIA, der Mossad etc. - seien daran interessiert, den dort befindlichen Eingang ins Erdinnere geheim zu halten. Der Vereinigung der getrennten arischen Völker und ihrer Zusammenführung mit der Urrasse am Pol habe der Krieg gegolten, den Hitler als Avatar - und zugleich als Oberhaupt der Geheimgesellschaft des schwarzen Ordens - geführt habe. Rudolf Heß' berühmter Flug nach Schottland im Jahre 1941 diente dem Versuch, die Deutschen mit ihren arischen Vettern, den Briten, wiederzuvereinen und auch diese zu einer Reinigung ihrer Rasse zu veranlassen.

Solche wüsten Phantasien greifen auf das bekannte Interesse einiger nationalsozialistischer Funktionäre an Hokuspokus und Geheimlehren zurück ${ }^{45}$ - es entbehrt nicht der Ironie, dass Ian Fleming, der spätere Autor der fames Bond-Romane, der im zweiten Weltkrieg im MI 6, dem britischen Auslandsgeheimdienst tätig war, allen Ernstes vorgeschlagen hatte, bei der Befragung von Rudolf Heß keinen geringeren als den berüchtigten Okkultisten Alastair Crowley als Vernehmungsoffizier einzusetzen, um der Verbindung von Nationalsozialisten mit obskurantistischen Kräften näher auf die Spur zu kommen.

Ergänzend sind in diesem Zusammenhang auch noch Spekulationen wie die von Jean Robin zu nennen, eines Theoretikers der Nouvelle Droite, der in seinem 1989 erschienenen Werk Opération Orth, ou l'incroyable secret de Rennes-le-Chateau, ${ }^{46}$ davon ausgeht, dass vom Queen-Maud-Land, das

44 Vgl. dazu Joscelyn Godwin, Arktos. Der polare Mythos zwischen NS-Okkultismus und moderner Esoterik, Graz 2007, S. 85.

45 Das berühmteste Beispiel für die okkulte Obsession der Nationalsozialisten bietet die Geschichte des jüdischen Hellsehers Hanussen. Vgl. dazu Wilfried Kugel, Hanussen - Die wahre Geschichte des Hermann Steinschneider, Düsseldorf 1998; vgl. auch Mel Gordon, Hanussen, Hitler's Jewish Clairvoyant, Port Townsend/Washington 2001.

46 Jean Robin, Opération Orth, ou l'incroyable secret de Rennes-le-Chateau, Paris 1989. 
von der deutschen Antarktisexpedition 1938/39 in Besitz genommen und in Neuschwabenland umgetauft wurde, eine direkte Verbindung zu einer unterirdischen Welt führt, in die Hitler sich gerettet habe. Allerdings ist er bei Robin nicht wie bei Serrano der Erlöser, der da kommen wird, sondern bereits 1953 gestorben: »Sein Körper wird in einem durchsichtigen sechseckigen Sarg aufbewahrt [...]." So schläft Hitler wie Schneewittchen einen tiefen Schlaf in der Hohlwelt, die zu einer Nekropole faschistischer Phantastereien verkommen ist. 
